\title{
Laser Speckle Imaging to Monitor Microvascular Blood Flow: A Review
}

\author{
Pedro G. Vaz, Student Member, IEEE, Anne Humeau-Heurtier, Edite Figueiras, Carlos Correia, \\ and João Cardoso
}

(Methodological Review)

\begin{abstract}
Laser speckle is a complex interference phenomenon that can easily be understood, in concept, but is difficult to predict mathematically, because it is a stochastic process. The use of laser speckle to produce images, which can carry many types of information, is called laser speckle imaging (LSI). The biomedical applications of LSI started in 1981 and, since then, many scientists have improved the laser speckle theory and developed different imaging techniques. During this process, some inconsistencies have been propagated up to now. These inconsistencies should be clarified in order to avoid errors in future works. This review presents a review of the laser speckle theory used in biomedical applications. Moreover, we also make a review of the practical concepts that are useful in the construction of laser speckle imagers. This study is not only an exposition of the concepts that can be found in the literature but also a critical analysis of the investigations presented so far. Concepts like scatterers velocity distribution, effect of static scatterers, optimal speckle size, light penetration angle, and contrast computation algorithms are discussed in detail.
\end{abstract}

Index Terms-Exposure time, gaussian distribution, laser speckle contrast imaging (LSCI), Lorentzian distribution, microvascular blood flow, static scatterersspeckle size.

\section{INTRODUCTION}

$\mathbf{T}$ HE laser speckle effect is a very useful physical phenomenon that is widely used in biomedical applications. Laser speckle is a random scattering phenomenon which can only be described statistically [1]. This effect occurs when coherent light is reflected by a rough surface on the scale of the used wavelength. This effect is characterized by a granular visual pattern composed by dark and bright spots.

The laser speckle effect was initially considered as a main drawback in the use of coherent light sources. The optical

Manuscript received November 6, 2015; revised January 15, 2016 accepted February 18, 2016. Date of publication February 24, 2016; date of current version September 16, 2016. This work was supported by the scholarship from Fundação para a Ciência e Tecnologia (SFRH/BD/89585/2012).

P. G. Vaz, J. Cardoso, and C. Correia are with the LIBPhys-UC, Department of Physics, University of Coimbra, Coimbra 3004-516, Portugal (e-mail: pvaz@lei.fis.uc.pt; jmrcardoso@uc.pt; correia@ fis.uc.pt).

E. Figueiras is with the International Iberian Nanotechnology Laboratory, Ultrafast Bio and Nanophotonics Group, Braga 4715-330, Portugal (e-mail: edite.figueiras@ inl.int).

A. Humeau-Heurtier is with the Laboratoire Angevin de Recherche en Ingénierie des Systèmes, University of Angers, Angers 49035, France (e-mail: anne.humeau@univ-angers.fr).

Digital Object Identifier 10.1109/RBME.2016.2532598 speckle limits the spatial resolution and decreases the signal-tonoise ratio (SNR) of multiple optical techniques, for example: optical coherence tomography, holography, laser projection systems, and synthetic aperture radar imagery [2]. The laser speckle pattern depends on the illumination source, the imaging system, and the imaged sample. If moving scatterers are present in the sample, then the speckle pattern changes along time. When this pattern is imaged with a finite exposure time, the areas of the image with moving scatterers will appear blurred (low contrast). The analysis of this blurring effect is the basis of laser speckle imaging (LSI) for biomedical applications, which can also be named laser speckle contrast imaging (LSCI).

The designation LSI is associated with a two-dimensional (2D) assessment technique used in many research fields and for many applications such as object velocity measurements [3], [4], surface roughness evaluation [5]-[7], laser vibrometry [8][10], and speech detection [11]. The biomedical applications of LSI will be discussed in more detail later in this study.

As pointed out by Humeau-Heurtier et al. [12], most part of biomedical laser speckle reviews are focused in only one part of the theoretical basis. In our opinion, a compendium about the methods that have been used since the year 1981 is missing in the literature. This review presents an overview of the laser speckle theory and practical concepts that can be found in the literature, and a critical analysis of these works. To understand the laser speckle nature and the methods used in biomedical applications, theoretical concepts like speckle contrast, decorrelation time, Lorentzian and Gaussian velocity models, and the effect of static scatterers will be explored herein. Moreover, an explanation of practical concepts such as speckle size, angle of incidence, and exposure time will be performed. Additionally, the most used image processing algorithms will be presented and, finally, the actual limitations of LSI will be discussed.

\section{SPECKLE THEORY}

Considering an observation plane, which is exposed to coherent light reflected from a scattering sample, the intensity registered in that plane, $I(t)$, at a specific point, is the coherent sum of many photon electric fields, $E(t)$, scattered from different points of the surface (or medium). Since each scattered wave has its own optical path, all the scattered waves arrive to the observation plane with phase differences leading to constructive or destructive interferences [13].

In perfect conditions, i.e., using a complete coherent and polarized light source and assessing a static medium, the resulting speckle pattern is fully developed, which means that their 


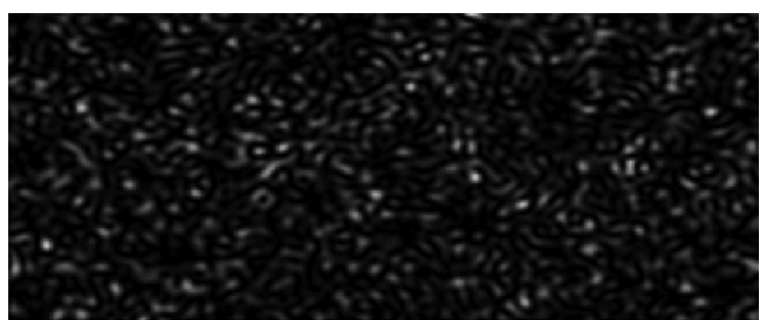

Fig. 1. Fully developed speckle pattern simulated using the algorithm proposed by Kirkpatrick et al. [14].

speckles are perfectly defined. An example of an ideal speckle pattern is presented in Fig. 1.

\section{A. Contrast}

It has been shown by Goodman [13] that, for a fully developed speckle pattern, there is an equality between its spatial standard deviation $\left(\sigma_{s}\right)$ and its spatial mean intensity $(\langle I\rangle)$. This useful property of the speckle interference effect is crucial for its application in the biomedical field [15]. Following this idea, the contrast $(K)$ is typically defined as the ratio between the standard deviation and the mean intensity [16]:

$$
K=\sigma_{s} /\langle I\rangle=\sqrt{\left\langle(I-\langle I\rangle)^{2}\right\rangle} /\langle I\rangle .
$$

Considering the statistical equality between $\sigma_{s}$ and $\langle I\rangle$ for a fully developed speckle pattern, we can easily conclude that the contrast of an ideal speckle pattern is 1 . In fact, under real conditions, the contrast is always lower than 1 and follows the condition $0 \leq K \leq 1$ [17].

In practice, the observed speckle pattern depends on the used coherent light source, on the illuminated medium, and on the image plane. The speckle pattern will remain unchanged if all these elements are static (static speckle pattern). On the other hand, a time change in one of these components causes a modification of the pattern (dynamic speckle pattern) [18].

If the scattering particles inside the medium are moving, the temporal pattern changes contain information about the motion of the particles. Two examples of these moving scatterers are the red blood cells flowing inside a blood vessel and the artery walls vibration. Imaging systems with finite exposure times perform an integration of all the light that reaches the sensor during the exposure time. If the speckle pattern changes during this integration time, the areas where the particles are moving become blurred [19].

It is clear that, by using an imaging system, it is the intensity $(I(t))$ of the speckle pattern that is recorded. The intensity spatial distribution (2-D image) can be used to compute the speckle contrast $(K)$. However, what is the relation between the measured contrast and the scatterers motion?

\section{B. Autocorrelation Functions}

Following the analysis performed by Boas and Yodh [20], we can deduce that the temporal fluctuations of the speckles intensity contain information concerning the dynamic properties of the medium. These intensity fluctuations are produced by electric field changes caused by the light scattered by moving particles. A good way to analyze these fluctuations is by using the normalized temporal autocorrelation function of the electric field which, for complex functions $(E(t) \in \mathbb{C})$, is [21]

$$
g_{1}(\tau)=\frac{\left\langle E(t) \cdot E^{*}(t+\tau)\right\rangle}{\left\langle E(t) \cdot E^{*}(t)\right\rangle}
$$

where $E(t)$ is the electric field over time $t, E^{*}$ denotes the complex conjugate of the electric field, $\tau$ the autocorrelation delay time, and \langle\rangle the time average. However, conventional imaging systems are not sensitive to the light electric field. They are sensitive to intensity fluctuations. Therefore, it is convenient to analyze the signal information as the normalized temporal autocorrelation function of the intensity [22]. Since the intensity is a real function $(I(t) \in \mathbb{R})$, its autocorrelation is simply,

$$
g_{2}(\tau)=\frac{\langle I(t) \cdot I(t+\tau)\rangle}{\left\langle I(t)^{2}\right\rangle} .
$$

The Siegert relation relates the second-order autocorrelation function [see (3)] with the first-order autocorrelation function [see (2)] [23], [24] as follows:

$$
g_{2}(\tau)=1+\beta\left|g_{1}(\tau)\right|^{2}
$$

where $\beta \leq 1$ is a normalization constant that accounts for the system imperfections, namely the absence of light stability and the speckle averaging due to spatial aliasing in the image detector [16], [25], [26]. In perfect conditions this constant is equal to 1 , and in the preliminary speckle studies it has been omitted [17], [19]. The additive value (1) results from the fact that the mean value of the intensity function is different from zero [20], contrary to the field mean value which is zero. The quadratic factor in (4) occurs because of the quadratic relation between amplitude and intensity [27].

It has been proven by Bandyopadhyay et al. [23] that the reduced variance of the intensity across the pixels is related with $g_{1}(\tau)$. This relation falls from the idea that the variance of the intensity of the speckle pattern is greater when the scatterers motion is slow compared to the imaging system exposure time. The following equation states the relationship between speckle contrast and the autocorrelation function in terms of the imaging system exposure time:

$$
K(T)^{2}=\frac{2 \beta}{T} \int_{0}^{T}\left|g_{1}(\tau)\right|^{2}\left(1-\frac{\tau}{T}\right) d \tau
$$

where $K(T)$ is the speckle contrast as function of the exposure time $T$. The second term in this expression $(1-\tau / T)$ was not used in the original expression of Briers and Webster [15] but several authors claim that this is the correct expression and must be used to obtain accurate results [25], [28]-[31]. This term falls from the transformation of the variance into the correlation where a triangular weighting must be multiplied to the autocorrelation function [23].

\section{Motion Distributions}

Two major types of motion can be considered for dynamic scatterers. These motions are classified as ordered motion (ordered flow of the scatterers) and disordered motion (Brownian 
motion) which occurs due to the effect of temperature [16]. The dynamic scatterers motion was historically considered to be close to Brownian motion [1], [16], [19], [29], [31]-[33] and with single scattering contribution [34]. This approach was used with the argument that the blood circulation is dense and turbulent [31]. The Brownian motion supports the approximation of the velocity distribution to a Lorentzian profile which leads to the field autocorrelation function:

$$
g_{1}^{L}(\tau)=e^{-\tau / \tau_{c l}}
$$

where $\tau_{c l}$ is the Lorentzian decorrelation time. The decorrelation time $\left(\tau_{c}\right)$ quantifies the dynamics of the scatterers [25]. In other words, lower values of $\tau_{c}$ are associated to scatterers with faster movements. Mathematically, this factor represents the autocorrelation function width, by determining the delay where the function falls from 1 to $1 / e$ [15]. The substitution of the autocorrelation function for the Lorentzian profile in (5) gives

$$
K^{L}(T)^{2}=\beta\left[\frac{\tau_{c l}}{T}+\frac{\tau_{c l}^{2}}{2 T^{2}}\left(e^{-2 T / \tau_{c l}}-1\right)\right] .
$$

The issue with this model is that, in fact, there are several motions present in blood flow [16], [35]. The ideal model can be explained as a combination of both Brownian (Lorentzian distribution) and ordered motion (Gaussian distribution). The autocorrelation function for a Gaussian velocity distribution, which is the correct one for ordered flow, has been proposed as [16]

$$
g_{1}^{G}(\tau)=e^{-\left(\tau / \tau_{c g}\right)^{2}}
$$

where $\tau_{c g}$ is the Gaussian decorrelation time. A different equation was suggested by Ramirez-San-Juan et al. [35] as the correct one to model a Gaussian velocity distribution. Their choice of the Gaussian model is based on the Mandel's definition of decorrelation time (see [35, Eq. (8)]) and can be described as

$$
g_{1}^{G}(\tau)=e^{-\frac{\pi}{2}\left(\tau / \tau_{c g}\right)^{2}} .
$$

In fact, (9) does not satisfy our original definition of the decorrelation time (falling from 1 to $1 / e$ in $\tau_{c}$ ). By opposition, (8) does meet this requirement and, additionally, it is the most referred in the literature to model a Gaussian velocity distribution. Having this into account, we assumed that (8) is the most correct one. By substituting the autocorrelation function for a Gaussian velocity profile, (8), in (5) we obtain

$$
\begin{aligned}
K^{G}(T)^{2}= & \beta\left[\frac{\tau_{c g}}{T} \sqrt{\frac{\pi}{2}} \operatorname{erf}\left(\sqrt{2} T / \tau_{c g}\right)\right. \\
& \left.+\frac{\tau_{c g}^{2}}{2 T^{2}}\left(e^{-2 T^{2} / \tau_{c g}^{2}}-1\right)\right] .
\end{aligned}
$$

It is important to note that the autocorrelation functions, (6) and (8), are valid for an approximation of single dynamic scattering regime but they present good agreement with the experimental results [34].

Some recent works [16], [22], [32] claim that biological tissues are complex systems with multiple sources of

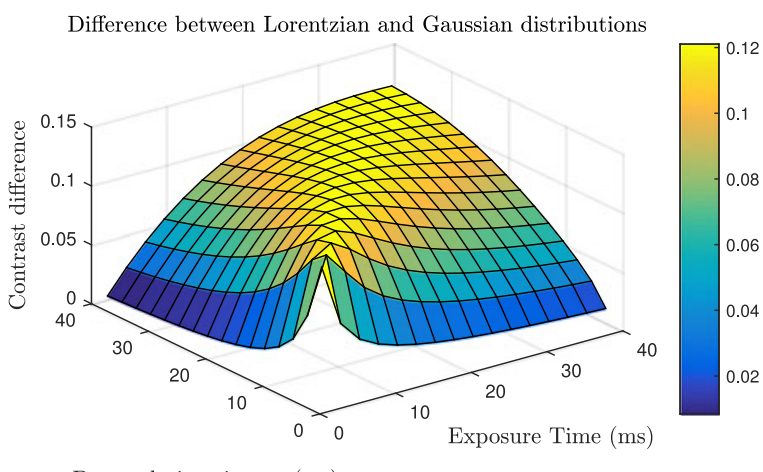

Decorrelation time $\tau_{c}(\mathrm{~ms})$

Fig. 2. Error in contrast between the use of Lorentzian and Gaussian distribution as function of the exposure time and decorrelation time.

decorrelation, i.e., multiple phenomenons that cause speckle pattern temporal changes. For this reason, an autocorrelation function that uses both Lorentzian and Gaussian distributions was proposed as an accurate model [22]

$$
\left|g_{1}^{L G}(\tau)\right|^{2} \approx\left|g_{1}^{L}(\tau)\right|^{2} \times\left|g_{1}^{G}(\tau)\right|^{2} .
$$

In the definition of this combined velocity distribution (Gaussian and Lorentzian) there is some misunderstandings because other authors [16] describe the model as a convolution between the two distributions yielding to a Voigt profile:

$$
g_{1}^{V}(\tau)=g_{1}^{L}(\tau) * g_{1}^{G}(\tau)
$$

where $g_{1}^{V}(\tau)$ represents the Voigt autocorrelation function.

The combined distribution improves the accuracy in the determination of the decorrelation time [16], [22]. However, the model that should be used for the autocorrelation function is still a major investigation field in LSCI [1]. Currently, the Lorentzian model is the most used in laser speckle studies [29], [33], [36][38] because the ordered flow is approximated by a Lorentzian distribution when scattering is random and uncorrelated [39]. Nevertheless, several authors [16], [40], [41] claim that the Gaussian distribution should be used.

Fig. 2 shows the difference in the contrast obtained when the function parameters ( $T$ and $\tau_{c}$ ) are fixed for both Lorentzian [see (7)] and Gaussian distributions [see (10)]. This figure shows that the largest differences, between the two profiles, are observed when the exposure time is equal to the laser speckle decorrelation time ( $T \approx \tau_{c}$ ). This is equivalent to say that the use of the Lorentzian distribution, to analyze ordered flows, yields to the largest errors when $\tau_{c} / T \approx 1$. This conclusion is particularly important because the sensitivity of speckle contrast, in relation to the decorrelation time $\left(\left|d K / d\left(1 / \tau_{c}\right)\right|\right)$, is maximum when $\tau_{c} / T=1$ [28], [42].

Equations (7) and (10) are, theoretically, the most correct ones, but some authors pointed that the use of the triangular weighting, as in (5)—second term, does not improve the results for biomedical applications when long exposure times $(T>>$ $\tau_{c}$ ) are used [16], [28], [43].

Smausz et al. [44] showed that the curve obtained using (5) without the triangular weighting can be approximated to the 


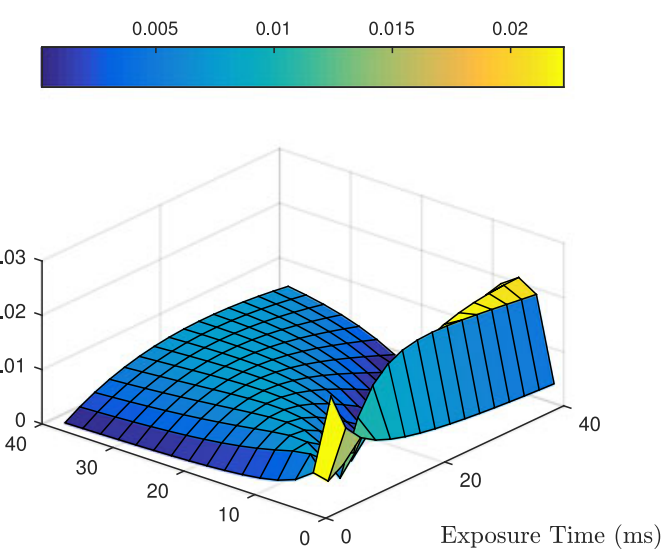

Decorrelation time $\tau_{c}(\mathrm{~ms})$

(a)

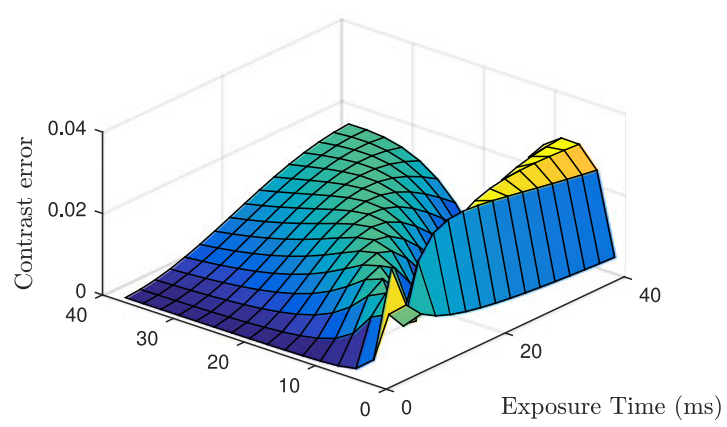

Decorrelation time $\tau_{c}(\mathrm{~ms})$

(b)

Fig. 3. Errors between the theoretical speckle visibility equations [see (7) and (10)] and the shifted approximated equations [see (13) and (14)]. (a) Lorentzian distribution. (b) Gaussian distribution.

theoretically correct one by substituting the variable $T / \tau_{c}$ by $\approx 0.57 \mathrm{~T} / \tau_{c}$ in the case of a Lorentzian distribution.

The steps to find this multiplier constant $\left(C_{s}=0.57\right)$ were not detailed in the work of Smausz et al., but the visual inspection of the two curves (triangular weighting versus variable substitution) shows only small differences between them (see [44, Fig. 3]). However, a qualitative approach is highly dependent on the window that is used to show the curves. The selected window has a great influence in the determination of $C_{s}$ that seems to lead to the best adjustment of both curves. In Smausz et al. work [44], the curves were plotted in a range of $0.001 \leq \tau_{c} / T \leq 10$.

In our opinion, a more numerical approach should be performed. We therefore performed a simulation in order to find which is the proper $C_{s}$ for both Lorentzian and Gaussian distributions. The $C_{s}$ was swapped between 0.1 and 0.9 with steps of 0.01 and the resultant function was compared with the theoretically correct ones [see (7) and (10)]. The comparison was performed by computing the root mean square (RMS) deviation between the two functions within a window of interest $\left(0.001 \leq \tau_{c} / T \leq 2\right)$. This window of interest was chosen because, in real applications, the exposure time is always higher than the scatterers decorrelation time. The lowest used exposure times are in the order of the millisecond, as it will be presented in Section III-E, but the decorrelation times are in the order of microsecond [25], [45].

The results of the simulation revealed that the constants which minimize the RMS deviation are: $C_{s}=0.61$ for the Lorentzian distribution with an error of $0.96 \%$; and $C_{s}=0.62$ for the Gaussian distribution with an error of $1.6 \%$. The approximated equations that correspond to (7) and (10) can thus be expressed as

$$
K^{L}(T)^{2} \approx \frac{\beta}{2} \frac{\tau_{c l}}{0.61 T} \times\left(1-e^{-2 \times 0.61 T / \tau_{c l}}\right)
$$

for a Lorentzian distribution and

$$
K^{G}(T)^{2} \approx \frac{\beta}{2} \frac{\tau_{c g}}{0.62 T} \sqrt{\frac{\pi}{2}} \times \operatorname{erf}\left(\sqrt{2} \times 0.62 T / \tau_{c g}\right)
$$

for a Gaussian distribution.
The differences between the theoretical equations and the approximated equations can be seen in Fig. 3. This figure shows that the error in the contrast determination is low for all the combinations of exposure times and decorrelation times. The maximum error, for the Lorentzian distribution, is 0.022 . For the Gaussian distribution it is 0.031 , which makes this approximation much more valid than the application of a Lorentzian distribution to ordered flow (see Fig. 2).

Approximated equations like (13) and (14) could be used when faster processing is required. These equations are much simpler and do not have any quadratic terms which reduces the computation time to evaluate them. Online video processing, in multiexposure speckle contrast techniques, takes a long time because it is necessary to fit the raw data to the selected equation for every single image pixel.

\section{Normalization Constants}

The normalization constant $(\beta)$ is linked with the experimental apparatus and needs to be computed for each individual system. The normalization constant can be computed as $\beta=\lim _{T \rightarrow 0} K(T)$ [46]. As stated in Section II-B, $\beta$ is a constant that accounts for the system light source instability, the system vibrations, nonperfect polarization of the laser light and, mainly, the spatial averaging that can occur when the speckle size is lower than two times the imaging system pixel size (Nyquist limit) [22].

Thompson et al. [26] and Zölei et al. [46] proposed a different normalization constant which is defined as $\beta_{m}=1 / K_{\max }$. This is equivalent to say that $\beta_{m}$ is the maximum contrast that the experimental system can achieve. This constant is multiplied by the measured contrast [left side of (5)]. Contrary, $\beta$ is a multiplication factor in the right side of (5). The definition of $\beta_{m}$ does not contradict the original definition of the normalization constant [20], [47] which states that $\beta \leq 1$. Although $\beta_{m}$ is always greater than $1\left(K_{\max }<1\right)$, it is multiplied in the opposite side of the equation which is in accordance with the original definition. 
Thompson et al. [26] also showed, by simulation and experiments, that $\beta_{m}$ is a correction factor that accounts for both spatial averaging and external illumination. In practice, since the normalization constant should be to "normalize" the contrast curve $(K(T))$, this second definition could be more useful and easier to determine experimentally.

Other types of normalization constant can also be used, in addition to $\beta$. These constants are often defined as a quantification of the noise present in the system [25] and are expressed as an additive term in (5). This term leads to its inclusion in all the equations that relate contrast, decorrelation time, and exposure time ((7), (10) and equivalents). In this review, we decide to omit this term in the theoretical analysis. Its influence will be discussed during the analysis of static scatterers and practical concepts (Sections II-E and III-D).

\section{E. Effect of Static Scatterers}

The presented models are based on a set of approximations that do not account for the presence of static scatterers in the assessed medium. However, static scatterers produce a speckle contrast component that remains constant when the imaging system exposure time increases. The light that is scattered by these types of elements cause an underestimation of the system dynamics. In other words, it increases the speckle contrast [28]. The static scatterers issue has been neglected in the past [23], [43], [48] but nowadays it is often accounted for [29], [34]. The existence of static scatterers has been addressed by Parthasarathy et al. [25] and Zakharov et al. [49] who presented a new method to compute accurate values of $\tau_{c}$.

In presence of static scatterers, the effective electrical field that reaches the imaging system is a sum of photons scattered by static particles and photons scattered by dynamic particles:

$$
E(t)=E_{d}(t)+E_{s}
$$

where $E_{d}(t)$ is the light electrical field scattered by dynamic particles and $E_{s}$ the light electrical field scattered by static particles which, since its contribution is static, does not vary with time [20], [24]. The new intensity autocorrelation function can be expressed as

$$
g_{2}(\tau)=1+\beta\left[(1-\rho)^{2}\left|g_{1 d}(\tau)\right|^{2}+2 \rho(1-\rho)\left|g_{1 d}(\tau)\right|+\rho^{2}\right]
$$

where $g_{1 d}(\tau)$ is the field autocorrelation function and $\rho$ is the fraction of light that is scattered by static particles, $\rho=$ $\left\langle I_{s}\right\rangle /\left(\left\langle I_{s}\right\rangle+\left\langle I_{d}\right\rangle\right)$. When using the constant $\rho$, one should be very careful about its definition. Inverse definitions can be found in the literature. For example, several authors define $\rho$ as the dynamic fraction of scattered light [22], [28], [40], [42] instead of the definition mentioned earlier.

The intensity autocorrelation function depends on the autocorrelation function of the dynamic component but does not depend on the autocorrelation function of the static component. This result is expected because, since the static electric field is constant, its autocorrelation function is approximately 1 for all the delay values $(\tau)[20]$.

The application of the Siegert relation, (4), to the intensity autocorrelation function yields to the modified field autocorrelation function [24]

$$
g_{1 m}(\tau)=(1-\rho)\left|g_{1 d}(\tau)\right|+\rho .
$$

By substituting $g_{1 m}$ in the integral of (5) we obtain

$$
\begin{aligned}
K^{2}(T)= & \frac{2 \beta}{T} \int_{0}^{T}\left[(1-\rho)^{2}\left|g_{1 d}(\tau)\right|+\rho\right]^{2}\left(1-\frac{\tau}{T}\right) d \tau \\
= & \frac{2 \beta}{T}\left[(1-\rho)^{2} \int_{0}^{T}\left|g_{1 d}(\tau)\right|^{2}\left(1-\frac{\tau}{T}\right) d \tau+2 \rho(1-\rho)\right. \\
& \left.\times \int_{0}^{T}\left|g_{1 d}(\tau)\right|\left(1-\frac{\tau}{T}\right) d \tau+\int_{0}^{T} \rho^{2}\left(1-\frac{\tau}{T}\right) d \tau\right] .
\end{aligned}
$$

The first integral is equivalent to (5). The modified speckle contrast shares a common part with (5) which exclusively depends on the dynamic autocorrelation. The other terms of (18) are dependent on the static autocorrelation.

The analytical result for the integral of (18), considering the autocorrelation function of the dynamic component as an exponential decay (Lorentzian distribution), is [28]

$$
\begin{aligned}
K(T)^{2}= & \beta\left[(1-\rho)^{2} \frac{e^{-2 x}-1+2 x}{2 x^{2}}\right. \\
& \left.+4 \rho(1-\rho) \frac{e^{-x}-1+x}{x^{2}}+\rho^{2}\right]
\end{aligned}
$$

where $x=T / \tau_{c}$. Using the same method, the speckle contrast for the modified autocorrelation function with Gaussian distribution is [22]

$$
\begin{aligned}
K(T)^{2}= & \beta\left[(1-\rho)^{2} \frac{e^{-2 x^{2}}-1+\sqrt{2 \pi} x \operatorname{erf}(\sqrt{2} x)}{2 x^{2}}\right. \\
& \left.+2 \rho(1-\rho) \frac{e^{-x^{2}}-1+\sqrt{\pi x} \operatorname{erf}(x)}{x^{2}}+\rho^{2}\right]
\end{aligned}
$$

Nadort et al. [22], [25], [26], [42] included in (19) and (20) an additive term that quantifies the noise present in the system ( $\nu_{\text {noise }}$ or $C_{\text {noise }}$ or $K_{0}$ or $P_{2}$ ). In practice, this term accounts for the minimum contrast that the system can achieve. This term is mainly used in analyses that take into account the presence of static scatters but it can also be applied in simpler studies.

We consider that (19) and (20), with or without the noise term, are the most complete equation to analyze laser speckle contrast data. These equations, known as the speckle visibility expressions [34], take into account the imperfections of the imaging systems, the noncomplete light coherence, and the presence of static scatterers in the medium. However, simpler results can be found in the literature [44], [46] as

$$
K(T)^{2}=\frac{1}{T} \int_{0}^{T} P_{1}^{2}\left|g_{1}(\tau)\right|^{2}\left(1-\frac{\tau}{T}\right)+P_{2}^{2} d \tau .
$$

This method includes two constants $\left(P_{1}\right.$ and $\left.P_{2}\right)$ in (5) that normalize the experimentally speckle contrast between 0 and 1 . These constants account for both the static scatterers and speckle spatial averaging, and can be determined by fitting this equation to the laser speckle data [46]. Even if this is a promising method that is very simple to apply, most of researchers still prefer 
using the methods described by Parthasarathy et al. [25] and Zakharov et al. [49], (18), because of their better relation with physics laws.

\section{F. Scatterers Velocity Determination}

The speckle contrast analysis starts with the computation of the imaging contrast and tries to determine the decorrelation time. The more rigorous is the decorrelation time determination, the more correct the scatterers velocity estimation will be. This is why so many different methods and analysis have been proposed during the last years, to improve the estimation of $\tau_{c}$. The most used relationship between scatterers velocity and decorrelation can be expressed as [15]

$$
V=\lambda /\left(2 \pi \tau_{c}\right)
$$

where $V$ is the scatterers velocity and $\lambda$ is the laser light wavelength. This relation does not have physics bases and is purely hypothetical [16], but it is the most used expression to relate the decorrelation time with the scatterers velocity. This simple relation is based on the intuitive notion that the velocity is inversely proportional to the decorrelation time $V \propto 1 / \tau_{c}$.

However, a different relation with a true physical meaning can be found in the literature [16], [32]

$$
V=\mathrm{PSF} / \tau_{c}
$$

where PSF is the point-spread function [32]. The PSF describes the response of an imaging system to a point source. From the knowledge of the authors, this velocity determination model has not been used in laser speckle contrast studies possibly due to the difficulty in determining the PSF.

The correct determination of $\tau_{c}$ and its relation with the velocity of the scatterers are the key factors to achieve a quantitative laser speckle analysis instead of a qualitative analysis, which is performed in most cases [28]. In laser speckle studies, arbitrary perfusion units are more used than velocity values because laser speckle has not proved yet to be a quantitative tool for complex systems [1], [16], [30], [31], [50].

This is in fact one of the most opened fields for research in LSCI. However, if the decorrelation time is computed with high accuracy, controlled phantoms or external methods can be used to calibrate laser speckle data [22], [34]. This issue will be discussed in more detail in Limitations and Future trends (see Section VII).

\section{Practical Considerations}

The theoretical considerations discussed in the previous section represent the basis of laser speckle contrast analysis (LASCA) for biomedical applications. However, researchers and engineers have to face another type of problem when performing experimental work or want to build laser speckle devices. In this case, the questions that rise are: How to maximize the speckle contrast? How to increase the SNR? What are the values for $\beta$ and $\rho$ ?

\section{A. Speckle Size}

One of the most important points to consider when designing a laser speckle system is the size of each individual speckle. When a digital imaging system is used, e.g., charge-coupled device (CCD) or CMOS sensors, its pixels have a finite size. When the size of each speckle, produced in the imaging sensor, is smaller than its pixel size, a spatial averaging occurs. In other words, more than one speckle is imaged by the same pixel which causes a decrease in contrast [12].

The speckle size in the imaging sensor depends on the wavelength $(\lambda)$ used and on the optical components used to image the pattern. The minimum resolvable speckle size, for a system using an imaging lens, is often expressed as [51]

$$
d_{\min } \approx 1.2(1+M) \lambda f / \#
$$

where $d_{\min }$ is the minimum speckle diameter in the same unit as the one used to express the wavelength, $M$ is the imaging system magnification, and $f / \#$ is the imaging system $\mathrm{f}$-number. From this expression, it is clear that the easiest parameter to control is the f-number which can be changed by using the iris diaphragm.

Thompson et al. [26] and Basak et al. [52] used (24) to compute an estimation of the speckle size. However, others [28], [31] used a different one $\left(d_{\min } \approx 2.4(1+M) \lambda f / \#\right)$. The oldest reference mentioning the size of speckle, from the best of our knowledge, can be found in Ennos's work [51]. This study states that the minimum diameter of a speckle, viewed by an imaging system, is given by (24). This expression is valid for the so-called "subjective" speckle [51]. The "subjective" speckle is obtained with imaging systems that use a lens to form the image in the sensor [53]. This type of speckle is the most common in biomedical applications.

It is also possible to use a different method to image the speckle pattern, the "objective" speckle [51]. This type of speckle is obtained as a projection of the pattern in the imaging sensor without any lens. In fact, the speckle pattern is a tri-dimensional field that propagates through the space. Therefore, the position of the imaging sensor in "objective" speckle will influence the observed pattern [54], [55].

Several authors studied the influence of different speckle sizes in the speckle contrast [26], [56], [57]. All the studies agree in the definition of speckle size as the number of pixels that a single speckle occupies in the imaging sensor (pixels/speckle). However, different optimal speckle sizes can be found in the literature. The first works on LSI tried to maximize the system spatial resolution by forcing a speckle size equal to the sensor pixel size. Also several recent papers [52], [58] continue to use $1 \mathrm{pixel} / \mathrm{speckle}$ which could be an unwise decision.

The work of Kirkpatrick et al. [57] showed, by simulation and by in vitro tests, that speckle sizes under the Nyquist limit lead to spatial aliasing, resulting in a reduction of up to $30 \%$ in the global contrast. They conclude that a minimum size of 2 pixels/speckle should be used for the speckle size. However, in our opinion, their results show that the contrast is still increasing for larger speckle sizes (up to 4 pixels/speckle). The increasing of the speckle size has the disadvantage of decreasing 


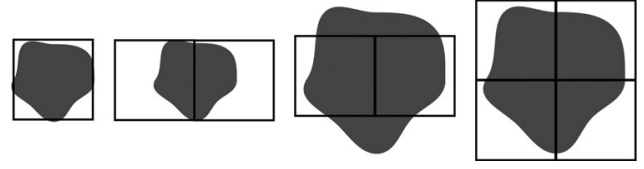

Fig. 4. Spatial sampling of speckles with different sizes. Each black square represents an imaging system pixel. The two left images represent a speckle size of $1 \mathrm{pixel} / \mathrm{speckle}$ and the two right images represent a speckle size of 2 pixels/speckle.

the spatial resolution because, since each speckle is the source of information, there are fewer speckles in the same area.

The simulations performed by Thompson et al. [26] showed that with 2 pixels/speckle there is also a significant decrease in the contrast (10\% lower than the theoretically maximum). However, the most important conclusion regarding speckle size, in our opinion, is that the optimal speckle size is influenced by the size of the window used to compute the contrast (see Section V).

Ramirez-San-Juan et al. [56] also addressed this issue and concluded that the speckle size has no strong influence in the determination of $\tau_{c}$. This result is more obvious when the temporal contrast (t-K) analysis is used (see Section V). However, this conclusion is only valid for relative measurements of $\tau_{c}$ in an homogeneous phantom. Their work is based on simplifications that are not valid in real cases, like the absence of static scatterers. Therefore, this result should be used carefully.

Contrary conclusions have also been reported. For example, Qiu et al. [59] suggested that speckle sizes under the pixel size can improve the SNR and the spatial resolution. This review also shows that $\beta$ can correct the speckle contrast even when the speckle size is under the pixel size. However, the metric used to analyze the SNR should be taken with caution (SNR $\equiv$ $\left.\mu_{K} / \sigma_{K}\right)$.

It is clear that when the speckle size is smaller than the pixel size, a spatial averaging occurs acting like a general 2-D lowpass filter. This filter produces an intensity increase in darker zones and an intensity decrease in brighter zones. The intensity mean is maintained but its standard deviation is reduced, leading to a reduction in the mean contrast and, more important, to the homogenization of the contrast values. This homogenization causes a reduction in the contrast standard deviation $\left(\sigma_{k}\right)$ which results in the increase of the SNR in the proposed metric.

The size defined by 2 pixels/speckle is also ambiguous since it is expected that each speckle has a circular shape. Fig. 4 illustrates the spatial sampling of speckles. The two images from the left show a speckle with diameter equals to one pixel. The two images from the right show a speckle with a diameter of 2 pixels/speckle. This illustration shows that it is necessary to have 4 pixels to sample a speckle with 2 pixels of diameter. In our opinion, considering a spatial contrast (s-K) computation (see Section V), the speckle size should be maintained above the Nyquist limit for both dimensions $(x$ and $y$ ). To ensure a correct sampling of a 2-D structure, at least two points should be sampled in each dimension. Further studies on the speckle size could lead to a conclusion in order to clarify the optimal speckle size.

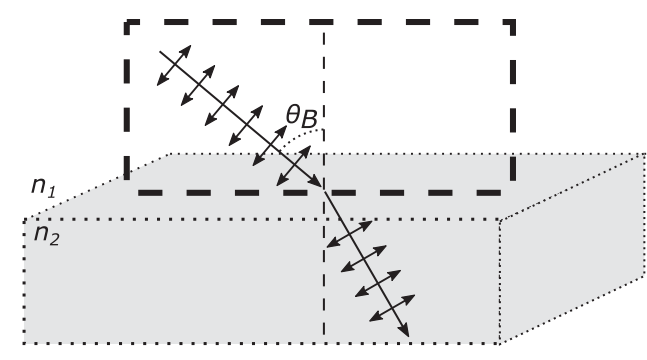

Fig. 5. Schematic representation of the Brewer's angle. $n_{1}$ and $n_{2}$ represent the refractive index of each medium and $\theta_{B}$ the Brewster's angle. The plane of incidence is represented by the thick-dashed rectangle.

The increase in the speckle size leads to a strong decrease in spatial resolution. First, less speckles are present in the same region and, second, larger processing windows must be used to ensure enough statistical relevance in the computation of the contrast. But, nowadays, imaging systems are very powerful and can have dozens of Megapixels which can balance the reduction of the resolution that happens due to the processing methods.

\section{B. Light Polarization}

The photons produced by light amplification by stimulated emission of radiation are coherent and, usually, linearly polarized. When light penetrates in a boundary between two media with distinct refractive indices $(n)$, some fraction of the light is reflected while the other fraction is transmitted. The amount of light that is reflected and transmitted depends on the angle of incidence of the photons, on its polarization, and on the media refractive indices. This light behavior is described by the Fresnel equations [60].

The light that is reflected in this process is partially polarized in the orientation perpendicular to the plane of incidence (spolarized). Most LSI systems use a polarizer in front of the imaging system to cut this light because it does not contain any information about the moving scatterers that are inside the tissue [52].

By the Fresnel equation, it is possible to prove that if the incident light is polarized parallel to the plane of incidence (ppolarized), there is an angle in which all the light is transmitted through the medium. This angle is the so-called Brewster's angle [61] and can be expressed as [60]

$$
\tan \left(\theta_{B}\right)=n_{2} / n_{1}
$$

where $\theta_{B}$ is the Brewster angle in radians, $n_{1}$ is the refractive index of the initial medium, and $n_{2}$ is the refractive index of the final medium. Fig. 5 shows the case when a p-polarized light beam reaches a media boundary with the Brewster's angle. There is no reflected beam because, as stated earlier, the reflected beam should be s-polarized but this component is not present in the incident beam.

From the knowledge of the authors, the Brewster's angle has not been explored in LSI systems for biomedical application. However, some authors have used an illumination angle between $30-40^{\circ}$ [39] and between $50-70^{\circ}$ [62], but with no estimation of the exact Brewster's angle. 
The use of this penetration angle could prevent the reflection of light in the first tissue layer, increasing the penetration depth and avoiding the use of the imaging system polarizer. This approach can also allow the reduction of the laser power since more light is transmitted to the tissue and interacts with the inner moving scatterers. However, the exact Brewster's angle is difficult to define because the optical refractive index of biological tissues is difficult to estimate. Moreover, since the samples are not usually flat, it is complicated to select an illumination angle very close to the Brewster's angle. These issues reduce the efficiency of this method. Anyway, more studies should be performed in order to confirm or refute this idea.

\section{Measuring Beta $(\beta)$}

The method used in laser speckle contrast theory-to account for the spatial averaging and partial light polarization-is the normalization constant $(\beta)$. The primary and the precise way to compute $\beta$ is to use the limit $\beta=\lim _{T \rightarrow 0} K(T)$. To compute this limit, it is necessary to take a set of contrast images with different exposure times $(T)$, from a unique dynamic sample $(\rho=0)$, and apply a fitting process to the obtained contrast [25]. The $y$-intercept value obtained by the fitting process is equal to $\beta$ and the function minimum value $\left(\lim _{T \rightarrow \infty} K(T)\right)$ is equal to $C_{\text {noise }}$.

A different process to compute $\beta_{m}$ (see Section II-D) was proposed by Thompson et al. [26] based on a single experimental measure. This process is interesting for single-exposure LSI systems. The experimental measurement consists in determining the maximum speckle contrast that the system can achieve by using a sample with only static scatterers $(\rho=1)$. In their work [26], the authors used a block of a mixture of silicone and alumina because this material tries to mimic the skin optical properties. Other authors have simply used a white sheet of paper to simulate the static scatterers [59]. The value of $\beta_{m}$ is then multiplied by the obtained contrast in order to normalize it.

\section{Measuring Rho $(\rho)$}

Even if the variable $\rho$ has been presented in many works as the correct way to account for static scatterers, its determination is not yet very clear. However, an experimental and objective method to compute $\rho$ has been presented by Zakharov et al. [24].

Typical LSI systems use exposure times $(T)$ longer than the dynamic scatterers decorrelation time $\left(\tau_{c}\right)$, and interframe times $(\Delta t)$ longer than the exposure times. Interframe time represents the time between the acquisition of one frame and the next one. Considering this, the relation $\Delta t>T>>\tau_{c}$ can be stated.

This relation proves that, between consecutive frames, all the dynamic scatterers have been completely decorrelated $\left(g_{1 d}(\Delta t) \approx 0\right)$ because their decorrelation time is much lower than the interframe time. Therefore, the correlation between consecutive frames appear only due to the presence of static scatterers. By substituting this proposition in (16) we obtain

$$
g_{2}(\Delta t)=1+\beta \rho^{2}
$$

where $g_{2}(\Delta t)$ is equivalent to the 2-D-correlation coefficient between two consecutive frames. Since the static scatterers distribution is not homogeneous in the tissues, the $\rho$ values should be computed locally, i.e., in regions of interest smaller than the image. The computation area is defined by the researcher. The initial proposition of this analysis invalidates its use when highspeed systems are used like the one proposed in [29] and [63]. This type of systems has interframes times much lower than the exposure time. In this case, a traditional analysis of the sample should be performed in advance to compute the values of $\rho$.

\section{E. Exposure Time}

The exposure time is another practical key factor that influences the final results of an LSI system. Different exposure times are sensitive to different scatterers velocity. For example, shorter exposure times are more suitable to analyze fast-moving scatterers [64]. This is a very intuitive notion because, at short exposure times, only rapid movements will cause blurring.

Some works have been published [42], [64] in order to determine the optimal exposure times to use in biomedical applications. Yuan et al. [64] proposed that, for single-exposure time LSI, its value should be $\approx 5 \mathrm{~ms}$. They showed that the relative sensitivity of speckle contrast is maximum when $T / \tau_{c}>1.8$ but also that the SNR decreases with the increase of exposure time. Their experiments dealt with cerebral blood flow (CBF). Single-exposure time systems can be found in the literature with many different exposure times. A tendency to use exposure times between 1 and $20 \mathrm{~ms}$ is noticed [65]-[70]. The most used LSI commercial devices are the moorFLPI (Moor Instruments-U. K.) and Pericam PSI (Perimed AB-Sweden). The exposure time of the moorFLPI can be set to $1,2,4$, or $8.3 \mathrm{~ms}$ [71]. Pericam PSI uses a fixed exposure time of $6 \mathrm{~ms}$.

Kazmi et al. [42] have performed an intensive study on the optimal exposure time and its optimization to decrease the acquisition time. The study started by using 15 exposure times ranging for $50 \mu$ s to $80 \mathrm{~ms}$ and the authors evaluated $\tau_{c}$ with a multiexposure speckle imaging (MESI). After that, the redundant exposure times were eliminated and a final optimal set of six exposure times was determined as $50 \mu \mathrm{s}, 0.25,0.75,5,25$, and $80 \mathrm{~ms}$.

This optimal set can be plotted as a logarithmic scale: short increases in the shorter exposure times and large increases in larger exposure times. This is an expected result because, for $\tau_{c}$ in physiological values, the $d K(T) / d T$ is higher for lower exposure times. In addition to these intensive studies, several exposure times ranges can be found in the literature, for example from $50 \mu$ s to $80 \mathrm{~ms}$ [22], [25], [34], from $50 \mu$ s to $30 \mathrm{~ms}$ [41], and from $200 \mu$ s to $20 \mathrm{~ms}$ [29].

As the exposure time becomes shorter, less photons are detected by the imaging sensor, which decreases the SNR. In conclusion, both too high and too short exposure times decrease the SNR, but for different reasons. Add to this: 1) the fact that to increase the speckle size the imaging iris must have a small aperture (some works reported the use of $f / 6$ [48] and $f / 4$ [72]), and 2) the polarizer that is often used in front of the imaging sensor reduces the number of detected photons. These facts 
make necessary the use of high light irradiance to illuminate the tissues in order to achieve acquisitions with short exposure times $(<1 \mathrm{~ms})$.

A pertinent question then arises: Can the optimal exposure time be dependent on the analyzed tissues? Most LSI applications are related with high perfused tissues (see Section VI) like the fore brain or the cornea. Additionally, most of the studies that try to optimize LSI are performed in this kind of tissues. In contrast, the analysis of skin, which is a much less perfused tissue, is also a major application of the LSI. Therefore, the optimization studies should also be performed for this kind of tissue.

\section{LSI TECHNIQUES}

Different LSI techniques have been developed since 1981. The LSI started with analog devices using only single-exposure times to assess the blood perfusion. In the 1980's, there was a well-established technique for blood perfusion assessment, the laser Doppler flowmetry (LDF). The research on laser speckle slowed down for many years due to its lack of performance compared with laser Doppler. However, the emergence of digital devices boosted the capabilities of LSI making it an attractive field of investigation.

\section{A. Single Exposure}

Single exposure LSI is a technique that is based on the computation of the laser speckle contrast [see (1)] by using one or more images acquired with a fixed exposure time. The contrast of these images is then related with the decorrelation time by a direct application of the expressions presented in Section II.

The pioneer work from Fercher and Briers [17], [19] proposed, for the first time, the use of laser speckle effect as a tool for microcirculation assessment. They used a photographic camera to image the retina during laser light illumination. The large exposure time (166 ms) and a completely analog imaging system caused problems to the technique despite its promising results.

The works of Fujii et al. [73], [74] were an improvement of the original technique because they used a linear CCD to image blood flow in the hands and fingers. This system required the use of a laser scanning system, composed of a moving mirror, in order to acquire perfusion maps (2-D blood flow images). Later, Tamaki et al. [75] used a CCD array to produce speckle images of the retina using a blue laser $(488 \mathrm{~nm})$. This time, the contrast was not computed spatially, as it has been done before, but it was computed temporally.

The milestone in the LSI occurred in 1996 with the work of Briers and Webster [15]. This paper proposed, and used for the first time, the acronym LASCA to define the analysis of laser speckle images using contrast computation for blood perfusion measurements. Even today, single-exposure LSI is widely used as a research tool because its results, although only quantitative, are conclusive and reliable [33], [65], [76], [77]. Single-exposure LSI has advantages over the traditional blood flow techniques (e.g., LDF), namely its capacity to perform flow maps (2-D images) at a reasonable cost.

\section{B. Multiexposure}

With the demand for a more quantitative LSI instrument, an improvement of the original technique emerged more recently [25]. This technique computes the contrast in multiple images, acquired with different exposure times, to perform a more accurate estimation of $\tau_{c}$. Consequently, several position vectors $(K, T)$ are computed leading to the possibility to represent the $K(T)$ function as a curve. Finally, this curve can be fitted using, for example, the least-squares method, and the parameter $\tau_{c}$ is extracted as the fitting variable.

The newer high-speed LSI devices [29], [63] adopt a different technique to produce multiexposure using only one exposure time. These systems can produce really high frame rates (up to $15 \mathrm{k}$ images/s) with low exposure times $(<70 \mu)$ and an interframe time so small that it can be neglected. The use of a highly sensitive imaging sensor, like an array of avalanche photo diodes, allows to emulate larger exposure times by simply summing the signals for each individual frame.

The current main line of research in the LSI technique is related to MESI systems [22], [26], [29], [30], [34], [46], [55], [78]. These custom systems have been developed by several researcher groups to perform a proof of concept and preclinical studies and their results are encouraging. Commercial devices only use single-exposure laser speckle [38]. However, the strong commercial interest of the MESI technique could conduct to a fast update of the existing devices or to the development of new imagers.

\section{CONTRAST COMPUTATION AlgORITHMS}

The evolution of the theory, instrumentation, and techniques was associated with the development of different ways to compute the laser speckle contrast. These methods can be classified into three categories: $\mathrm{s}-\mathrm{K}, \mathrm{t}-\mathrm{K}$, and, a combination of both, spatio-temporal (st-K) [18].

\section{A. Spatial Contrast}

The s-K was used in the work of Briers and Webster [15] and consists in computing the contrast in small regions (elements) of the laser speckle raw image. Normally, these elements correspond to squares of $3 \times 3$ [12], $5 \times 5$ [48], [79] or $7 \times 7$ [15] pixels. The general equation to compute $s-\mathrm{K}$ in raster images can be expressed as [18]

$$
\begin{aligned}
\mu_{i, j, t}^{s}= & \frac{1}{n^{2}} \sum_{x=i-\frac{n-1}{2}}^{i+\frac{n-1}{2}} \sum_{y=j-\frac{n-1}{2}}^{j+\frac{n-1}{2}} I_{x, y, t}, \\
K_{i, j, t}^{s}= & \frac{\sqrt{\frac{1}{n^{2}} \sum_{x=i-\frac{n-1}{2}}^{i+\frac{n-1}{2}} \sum_{y=j-\frac{n-1}{2}}^{j+\frac{n-1}{2}}\left(I_{x, y, t}-\mu_{i, j, t}^{s}\right)^{2}}}{\mu_{i, j, t}^{s}}
\end{aligned}
$$

where $\mu_{i, j, t}^{s}$ is the mean intensity value in the element with a central pixel in the coordinates $(i, j, t), n$ is the lateral size of the element, and $I_{x, y, t}$ is the raw speckle value in the respective coordinates. Finally, $K_{i, j, t}^{s}$ is the contrast value associated with 


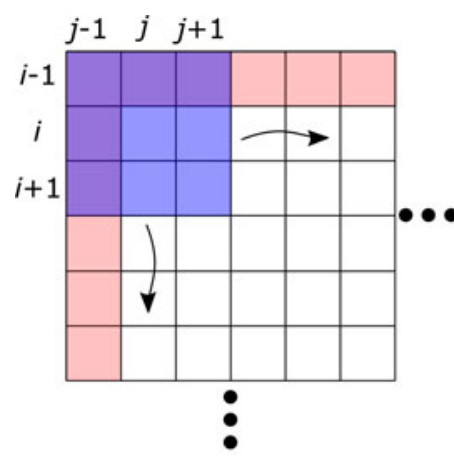

(a)

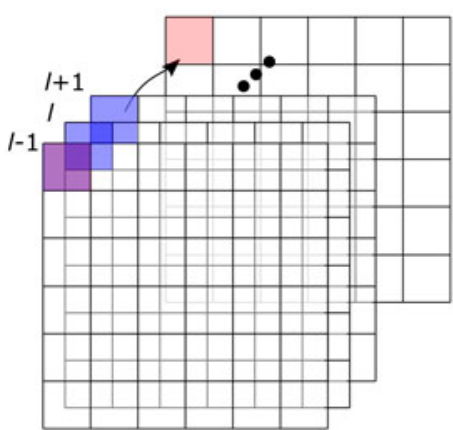

(b)

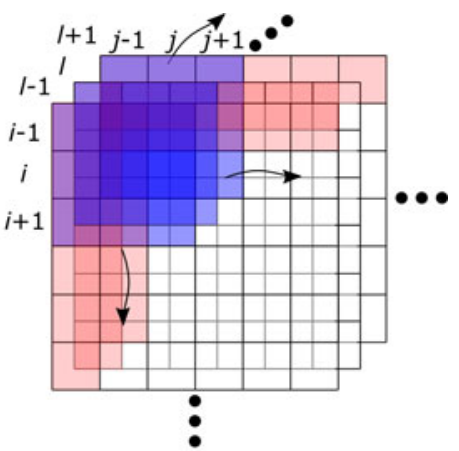

(c)

Fig. 6. Illustration of the elements to compute contrast. The element is represented by blue squares. Pink squares represent the boundaries where the computation of contrast requires padding. Curved arrows were used to indicate the element displacement directions. (a) s-K. (b) t-K. (c) st-K.

the element centered in $(i, j, t)$. Attention must be given to the boundary of the image since it is impossible to evaluate $(I)$ at negative coordinates. Two possible solutions can be applied: 1 ) exclude the outer borders of the image from the analysis or 2) using padding techniques for the outer borders of the images.

Fig. 6(a) illustrates an s-K element with size $3 \times 3$ pixels (blue square) with a central pixel $(x, y, t)=(i, j, 1)$. The pixels colored in pink represent the image boundary in which the contrast computation can only be computed using padding techniques. This boundary depends on the element size and is typically $(n-1) / 2$ rows and columns. The s-K element is then moved along the $x$ - and $y$-directions to compute a contrast 2-D map. The element displacement is often used without overlapping.

Laser speckle contrast is a statistic measure because it is related to the mean and the standard deviation of the speckle image intensity [see (1)]. Therefore, the larger the element, i.e., the processing region, the better the contrast estimation. However, the increase of the element size causes a decrease in the spatial resolution of the contrast images and, therefore, of the perfusion map. Larger sizes can be used when high-resolution laser speckle images are acquired and the spatial resolution is not a key factor.

To select a correct element size, it is necessary to take into account the speckle size present in the raw images (see Section III-A). To estimate the contrast in a statistically relevant number of speckles, the element size should be proportional to the speckle size. Consequently, when the value of pixels/speckle is high, larger elements should be used. Duncan et al. [80] studied the relation between speckle size and element size but their analysis, in our opinion, is not clear about the relation between speckle size and the optimal element size.

\section{B. Temporal Contrast}

Another type of algorithm was proposed by Cheng et al. [79] in order to improve the spatial resolution. The $\mathrm{t}-\mathrm{K}$ is computed by using a set of statistically independent frames. These frames can only be consecutive when the interframe times $(\Delta t)$ is large enough which, as stated early, does not occur in high-speed systems [29], [63]. The minimum number of frames $(m)$ that should be used, in the computation of the $\mathrm{t}-\mathrm{K}$, to obtain a valid statistic is 15 [43]. The maximum number of frames only depends on the minimum admissible temporal resolution but references can be found that use up to 49 frames [18]. The t-K can be computed using [18]

$$
\begin{aligned}
\mu_{x, y, l}^{t} & =\frac{1}{m} \sum_{t=l-m / 2}^{l+m / 2} I(x, y, t) \\
K_{x, y, l}^{t} & =\frac{\sqrt{\frac{1}{m} \sum_{t=l-m / 2}^{l+m / 2}\left(I_{x, y, t}-\mu_{x, y, l}^{t}\right)^{2}}}{\mu_{x, y, l}^{t}}
\end{aligned}
$$

where $\mu_{x, y, l}^{t}$ is the temporal mean of the element with central pixel in $(x, y, l)$ and size $1 \times 1 \times m$ pixels, $K_{x, y, l}^{t}$ is the $\mathrm{t}-\mathrm{K}$ within this element.

Fig. 6(b) illustrates the application of $\mathrm{t}-\mathrm{K}$ algorithm with an element of size $1 \times 1 \times 3$ and centered in the pixel $(x, y, t)=$ $(1,1, l)$. This element is displaced along the temporal direction $(t)$ until the last frame. Subsequently, this element is moved to another pixel, i.e., to another location in the first image.

The $\mathrm{t}-\mathrm{K}$ reduces the temporal resolution while the s-K reduces the spatial resolution. To select the suitable algorithm, it is important to have in mind which dimension (spatial or time) is the most important to preserve. Additionally, $\mathrm{t}-\mathrm{K}$ proved to accurately estimate contrast in the presence of static scatterers [81]. Ramirez-San-Juan et al. [82] recently showed in vitro that the values computed with the $\mathrm{t}-\mathrm{K}$ algorithm are independent of the static scatterers layer thickness.

The use of $\mathrm{t}-\mathrm{K}$ has increased due to the advantage of dealing with static scatterers [25], [83]. In our opinion, temporal laser speckle contrast should be applied in the case of a speckle size equal to the pixel size. The use of $\mathrm{t}-\mathrm{K}$ with larger speckle size (e.g., 2 pixels/speckle) will cause a decrease in both the spatial and temporal resolutions without improving the $\mathrm{t}-\mathrm{K}$ values [25]. Nevertheless, one study [56] suggests that the increase of speckle size causes an increase in speckle contrast for both spatial and temporal algorithms.

We would like to propose the hypothesis that, using fast imaging systems, the $\mathrm{t}-\mathrm{K}$ can be used for very short exposure times $(T \approx 50 \mu \mathrm{s})$. By this method, the $\mathrm{t}-\mathrm{K}$ information will come 
from the time variations of a single pixel, and not from the "blurring" of an image region. The shorter the exposure time, the better the speckle sampling will be. In the case of short exposure times, a static speckle will appear with approximately the same intensity along several images, leading to low $\sigma$ and low contrast. Additionally, a moving speckle will appear with different intensities due to changes in the interference of the light leading to high $\sigma$ and high contrast. This is exactly the inverse of the s-K. This new interpretation of the contrast could be related with the scatterers decorrelation time. This hypothesis falls from the Nyquist theorem which states that signals must be sampled with, at least, twice their maximum frequency. Further studies to refute or confirm this hypothesis need to be conducted.

\section{Spatio-Temporal Contrast}

A combination of both spatial and temporal algorithms can be applied to compute the speckle contrast [84]-[87]. The used element is usually a cuboid [see Fig. 6(c)] that can be isotropic or anisotropic. The expression for the st-K computation is not presented due to page limitation but its deduction is trivial.

Fig. 6(c) illustrates the application of the st-K with a cubic element with size $3 \times 3 \times 3$ pixels and centered in the pixel $(x, y, t)=(i, j, l)$. This element is displaced in the spatial and temporal dimensions. Different sizes of elements can be used to compute st-K. For example, Duncan and Kirkpatrick [85] used an element size of $3 \times 3 \times 15\left(N_{x}, N y, N_{t}\right)$ pixels. Usually, anisotropic elements are used in which the spatial dimensions are equal and the temporal dimension is different. The temporal dimension of the element is usually larger than the spatial dimensions to ensure good statistical time evaluation.

Qiu et al. [86] studied the effect of the temporal dimension of the element. They used elements with spatial dimensions of $3 \times 3$ and $9 \times 9$ pixels and a temporal dimension range from 3 to 30 pixels. This study found that the mean value of the contrast $\left(\mu_{s t}\right)$ depends on both the spatial dimensions and the temporal dimension and, for practical applications, a $3 \times 3 \times 15$ pixels (anisotropic) or a $5 \times 5 \times 5$ (isotropic) pixels elements are recommended.

Alternatively, Rege et al. [84] proposed a different approach for anisotropic st-K. Their method uses an element with the same orientation as the analyzed blood vessel with dimensions of $9 \times$ $1 \times 3$ pixels for $N_{x}, N y$, and $N_{t}$, respectively. The requirement of only three temporal frames increases the temporal resolution of this method compared with the standard elements used in st-K.

\section{BiomedicAl Applications}

Multiple biomedical applications can be found for LSI. This section is a short compilation of some practical studies and their conclusions. A more extensive review on applications can be found in [1], [30], [31], [36], [77], [88].

\section{A. Microcirculation-Blood Perfusion}

Historically, LSI was used to assess blood perfusion and blood flow, mainly for retinal and cerebral applications (CBF). These are the perfect applications for LSI because the blood vessels are close to the tissue surface leading to high SNR.

LSI was used to study the influence of collateral blood flow during an ischemic stroke in rats [89]. A single-exposure LSI ( $T=15 \mathrm{~ms}$ ) was used with a spatial speckle contrast computed from elements of $5 \times 5$ pixels. The authors reported that, after the vessel occlusion, extensive anastomatic connections were formed to augment blood flow. Moreover, it has been shown that, using single-exposure LSI $(\mathrm{T}=20 \mathrm{~ms})$, hyperglycemia worsens the effect of a cerebral stroke in rats [90]. A LSI system, with fixed exposure time $(T=5 \mathrm{~ms})$, has been used during human neurosurgery in order to monitor the CBF. This system allows to represent online perfusion maps without interference with the surgery [91]. Additionally, MESI (exposure times 1$100 \mathrm{~ms}$ ) was used to study the cortical microcirculation of piglets during the application of various vasodilators [92]. Finally, a dual wavelength LSI was used to assess brain hemodynamics and CBF [93].

Retinal blood analyses is also a major application of an LSI. A single-exposure LSI ( $T=40 \mathrm{~ms}$ ) has been used to image the retinal blood flow [94] during light stimulation. It has been found that flickering light dilated retinal arterioles and increases retinal blood velocity. Furthermore, changes in retinal blood flow according to the position (sitting or supine) were also studied using LSI [95]. Change from a sitting position to a supine position causes an increase in blood flow during 6 min. Moreover, Ponticorvo et al. [96] have combined single-exposure LSI ( $T=$ $5 \mathrm{~ms}$ ) with an endoscope to analyze retinal blood flow changes in rats. They concluded that the blood flow increases during visual stimulus and hypercapnia while it decreases during hyperoxia.

Skin perfusion assessment represents another research field in which LSI is widely used. Rege et al. [72] used a single-exposure LSI $(T=16 \mathrm{~ms})$ to assess the microvascular remodeling during wound healing. They found that microvessel density increases in the initial tissue inflammation and returns to baseline during the remodeling stage. Moreover, others have detected lower levels of peripheral blood perfusion in systemic sclerosis patients compared with healthy subjects, using single-exposure LSI ( $T=6 \mathrm{~ms}$ ) [97], [98]. Domsic et al. [99] found that the early endothelial changes occur in the small arterioles and capillaries of early systemic sclerosis patients. Furthermore, LSI has been used to assess the microvascular function in primary Raynaud's phenomenon and atherosclerosis [100], [101]. Finally, Cordovil et al. [102] used LSI to assess the skin of the forearm and found that, in cardiometabolic disease patients, the vasodilator responses were significantly reduced.

\section{B. Macrocirculation-Blood Pressure}

Apart from the original applications of LSI in blood flow measurements, new applications in the assessment of macrocirculation begin to emerge. LSI has been used to remotely estimate the blood pulse pressure waveform in the radial artery [2], [103], [104], carotid artery [105], and tooth [106]. Additionally, LSI was used to detect the blood pulsation in presence of motion artifacts [107]. Measurements were performed in the finger and images were processed using contrast and 
correlation. Finally, CBF in rats was synchronized with cardiac cycle using LSI [66]. This synchronization allows the correction of pulsation-modulated speckle contrast signals.

\section{LIMITATIONS}

Laser speckle has proven to be a simple and very versatile tool that can be applied in many fields of investigation and clinical studies. However, two major limitations of LSI systems need to be addressed.

\section{A. Absolute Velocity Estimation}

Interstudies evaluation is impossible to perform when qualitative laser speckle methods are used. This occurs because LSI acquisition protocols do not follow a standard and only arbitrary perfusion units or percentage variations are analyzed.

The absolute velocity of scatterers is extremely difficult to measure and depends on several factors [16], [30], [108]. These factors have been discussed above and include the nonperfect light polarization, multiple scattering, static scatterers, speckle spatial averaging, approximated velocity models, and the relation between velocity, and decorrelation time. However, Li et al. [62] claim to achieve direct measurements of the scatterers velocity with a frequency domain LSI (FDLSI) technique. This technique is based on the determination of $\tau_{c}$ using the Fourier transform of the intensity, similarly to the laser Doppler technique. The results from FDLSI are promising and should be followed.

The influence of the blood vessel caliber in the $\tau_{c}$ determination is also a major issue in the absolute velocity estimation and it has been studied by some authors [34]. The probability of having multiple scattering is higher with large caliber blood vessels compared with small caliber blood vessels [109]. The occurrence of different scattering degrees may cause a poor estimation of $\tau_{c}$. In Kazmi et al. work [34], they suggest that $\tau_{c}$ is proportional to the product between the blood velocity and the blood vessel diameter. Moreover, the scatterers concentration has impact on the estimated contrast, but only in lower ranges [110].

Based on these grounds, LSI is represented in arbitrary units which can have different definitions. These arbitrary units can be expressed as the contrast value, the decorrelation time $\left(\tau_{c}\right)$ [36], the speckle flow index [67], the inverse correlation time [34], the relative flow volume [111], and the percentage of the baseline [76]. Different techniques to achieve an estimation of red blood cells velocities have been proposed by using calibration with phantoms [62], [112] or other systems [22], [113], [114].

To solve this issue, future works should be focused on the improvement of theoretical models. Accounting for the multiple scattering that occurs in biological tissues, clarifying the relation between decorrelation time and scatterers velocity, and approaching the experimental conditions to the theoretical assumptions are essential subjects to be studied [77].

\section{B. Deep Assessment}

The second limitation of LSI is related with the maximum depth that produces laser speckle contrast information.
According to some authors [115], the penetration of the photons in LSI standard techniques is about $300 \mu \mathrm{m}$. Alternative techniques, like LDF, can achieve assessment depths up to about $900 \mu \mathrm{m}[116]$.

To improve the penetration depth of LSI several studies have been performed recently [65], [109], [117]-[120]. These studies present novel LSI techniques, in most of the cases with hardware changes. For example, He et al. [118] used a linear laser scanning system to collect speckle images. In each image, only the zones distant from the laser illumination are used to compute the contrast image. The light that is refracted in areas farther from the penetration zone reaches deeper tissues. This is the socalled "banana path" [65], [121]. This technique takes advantage of the same principle as diffuse correlation spectroscopy [122] Bi et al. [117] have used the same principle but with a point-like laser light source that illuminates the tissue outside the imaging system field of view. The pixels, in the imaging system, farther from the illumination point record photon arising from deeper tissues. Finally, Varma et al. [65] proposed a speckle contrast optical tomography that can render 3-D volumes of flow information.

These types of techniques work in a complete multiscattering regime, which, in our opinion, may limit the use of the theoretical analysis presented in Section II because it assumes a single dynamic scattering regime. More studies should be conducted to conclude if the single scattering approximations performed in the laser speckle contrast theory are valid even in these new LSI techniques.

\section{CONCLUSIONS AND FutURE TRENDS}

LSI is a powerful technique that is evolving fast both in the application of new theories and the development of new instruments. This review presented a critical analysis of the LSI principles and a clarification of some ideas, that we believe, are not well explained nor compiled in the available literature.

The resolution of the limitations presented in Section VII are two of the future trends in LSI. Additionally, Humeau-Heurtier et al. [77] point the possibility to perform blood perfusion measurements in moving subjects. This could be a major advance because vascular diseases are often displayed during exercise and in patients having involuntary movements, e.g., Parkinson disease.

Furthermore, the combination of LSI data with other micro/macro circulation assessment techniques could improve the diagnosis of vascular diseases. The application of automatic classifiers like neural network or support vector machine to features extracted from multiple information sources have been applied in other areas [123]. This could help improving diagnosis and the SNR of LSI.

Moreover, MESI is a recent technique (2008) which is only available in prototypes. Several questions and improvements, which were detailed in this review, are still possible for MESI. Among them we can cite the establishment of standards in order to make possible the comparison of results in studies performed with different laser speckle systems [77]. 
The clarification of subjects like: 1) the approximations behind the laser speckle theory, 2) the differences between the Lorentzian and Gaussian motion models, 3) the optimal speckle size for each processing algorithms, 4) the experimental processes to compute the normalization constant $\beta$ and the static scatterers coefficient $\rho$, and (5) the influence of the exposure time, was performed in this review.

The technical advantages of LSI improve the realization of clinical studies in microcirculation. The possibility to obtain 2-D perfusion maps with high frame rates and at reasonable cost is a key factor to make this assessment technique highly competitive. Because of these advantages, LSI has been widely used in biomedical applications but much more fields will certainly be explored in the future.

\section{REFERENCES}

[1] D. Briers, D. Duncan, S. Kirkpatrick, M. Larsson, T. Stromberg, and O. Thompson, "Laser speckle contrast imaging: Theoretical and practical limitations," J. Bomed. Opt., vol. 18, no. 6, pp. 1-9, 2013.

[2] P. Vaz, P. Santos, E. Figueiras, C. Correia, A. Humeau-Heurtier, and J. Cardoso, "Laser speckle contrast analysis for pulse waveform extraction," Proc. SPIE, vol. 9540, pp. 954006-954007, 2015.

[3] T. Asakura and N. Takai, "Dynamic laser speckles and their application to velocity measurements of the diffuse object," Appl. Phys., vol. 194, pp. 179-194, 1981

[4] P. Šmíd, P. Horváth, and M. Hrabovský, "Speckle correlation method used to measure object's in-plane velocity," Appl. Opt., vol. 46, no. 18, pp. 3709-3715, 2007.

[5] H. Hirabayashi, T. Matsuo, H. Ishizawa, H. Kanai, and T. Nishimatsu, "Surface roughness evaluation by laser speckle," in Proc. Int. Joint Conf. SICE-ICASE, 2006, pp. 5809-5812.

[6] A. M. Hamed, H. El-Ghandoor, F. El-Diasty, and M. Saudy, "Analysis of speckle images to assess surface roughness," Opt. Laser Technol., vol. 36, no. 3, pp. 249-253, Apr. 2004.

[7] T. Yoshimura, K. Kato, and K. Nakagawa, "Surface-roughness dependence of the intensity correlation function under speckle-pattern illumination," J. Opt. Soc. Amer. A, vol. 7, no. 12, pp. 2254-2259, 1990.

[8] S. Rothberg and B. Halkon, "Laser vibrometry meets laser speckle," Proc. SPIE, vol. 5503, pp. 280-291, 2004.

[9] P. Martin and S. Rothberg, "Introducing speckle noise maps for laser vibrometry,” Opt. Lasers Eng., vol. 47, nos. 3/4, pp. 431-442, 2009.

[10] A. A. Veber, A. Lyashedko, E. Sholokhov, A. Trikshev, A. Kurkov, Y. Pyrkov, A. E. Veber, V. Seregin, and V. Tsvetkov, "Laser vibrometry based on analysis of the speckle pattern from a remote object," Appl. Phys. B, vol. 105, no. 3, pp. 613-617, Jun. 2011.

[11] Z. Zalevsky, Y. Beiderman, I. Margalit, S. Gingold, M. Teicher, V. Mico, and J. Garcia, "Simultaneous remote extraction of multiple speech sources and heart beats from secondary speckles pattern." Opt. Exp., vol. 17, no. 24, pp. 21566-21580, Nov. 2009.

[12] A. Humeau-Heurtier, P. Abraham, and G. Mahe, "Linguistic analysis of laser speckle contrast images recorded at rest and during biological zero: Comparison with laser doppler flowmetry data," IEEE Trans. Med. Imag., vol. 32, no. 12, pp. 2311-2321, Dec. 2013.

[13] J. Goodman, "Statistical properties of laser speckle patterns," in Laser Speckle Relat. Phenom., ser. Topics in Applied Physics, vol. 9. Berlin: Germany: Springer, 1975, pp. 9-75.

[14] S. Kirkpatrick, D. Duncan, R. Wang, and M. Hinds, "Quantitative temporal speckle contrast imaging for tissue mechanics." J. Opt. Soc. Amer. A. Opt. Image Sci. Vis., vol. 24, no. 12, pp. 3728-3734, 2007.

[15] J. D. Briers and S. Webster, "Laser speckle contrast analysis (LASCA): A nonscanning, full-field technique for monitoring capillary blood flow," J. Biomed. Opt., vol. 1, no. 2, pp. 174-179, 1996.

[16] D. D. Duncan and S. J. Kirkpatrick, "Can laser speckle flowmetry be made a quantitative tool?" J. Opt. Soc. Amer. A. Opt. Image Sci. Vis., vol. 25, no. 8, pp. 2088-2094, Aug. 2008.

[17] J. D. Briers and A. F. Fercher, "Retinal blood-flow visualization by means of laser speckle photography," Invest. Ophthalmol. Vis. Sci., vol. 22, no. 2, pp. 255-259, 1982.
[18] M. Draijer, E. Hondebrink, T. van Leeuwen, and W. Steenbergen, "Review of laser speckle contrast techniques for visualizing tissue perfusion," Lasers Med. Sci., vol. 24, no. 4, pp. 639-651, 2009.

[19] A. F. Fercher and J. D. Briers, "Flow visualization by means of singleexposure speckle photography," Opt. Commun., vol. 37, no. 5, pp. 326330, 1981.

[20] D. Boas and A. G. Yodh, "Spatially varying dynamical properties of turbid media probed with diffusing temporal light correlation," J. Opt. Soc. Amer. A, vol. 14, no. 1, pp. 192-215, 1997.

[21] G. Yu, T. Durduran, C. Zhou, R. Cheng, and A. G. Yodh, "Near-infrared diffuse correlation spectroscopy for assessment of tissue blood flow," in Handbook of Biomedical Optics, 1st ed. Boca Raton, FL, USA: CRC Press, 2011, ch. 10, pp. 195-216.

[22] A. Nadort, R. G. Woolthuis, T. G. van Leeuwen, and D. J. Faber, "Quantitative laser speckle flowmetry of the in vivo microcirculation using sidestream dark field microscopy," Biomed. Opt. Exp., vol. 4, no. 11, pp. 2347-2361, 2013.

[23] R. Bandyopadhyay, A. S. Gittings, S. S. Suh, P. K. Dixon, and D. J. Durian, "Speckle-visibility spectroscopy: A tool to study timevarying dynamics," Rev. Sci. Instrum., vol. 76, no. 9, p. 93110, 2005.

[24] P. Zakharov, A. C. Völker, M. T. Wyss, F. Haiss, N. Calcinaghi, C. Zunzunegui, A. Buck, F. Scheffold, and B. Weber, "Dynamic laser speckle imaging of cerebral blood flow," Opt. Exp., vol. 17, no. 16, pp. 13904$13917,2009$.

[25] A. B. Parthasarathy, W. J. Tom, A. Gopal, X. Zhang, and A. K. Dunn, "Robust flow measurement with multi-exposure speckle imaging," Opt. Exp., vol. 16, no. 3, pp. 1975-1989, Feb. 2008.

[26] O. Thompson, M. Andrews, and E. Hirst, "Correction for spatial averaging in laser speckle contrast analysis," Biomed. Opt. Exp., vol. 2, no. 4, pp. 1021-1029, Apr. 2011.

[27] N. C. Santos and M. A. Castanho, "Teaching light scattering spectroscopy: The dimension and shape of tobacco mosaic virus," Biophys. J., vol. 71, no. 3, pp. 1641-1650, 1996.

[28] D. A. Boas and A. K. Dunn, "Laser speckle contrast imaging in biomedical optics," J. Biomed. Opt., vol. 15, no. 1, pp. 011109-1-011109-12, 2010.

[29] T. Dragojević, D. Bronzi, H. Varma, C. Valdes, C. Castellvi, F. Villa, A. Tosi, C. Justicia, F. Zappa, and T. Durduran, "High-speed multiexposure laser speckle contrast imaging with a single-photon counting camera," Biomed. Opt. Exp., vol. 6, no. 8, pp. 2865-2876, 2015.

[30] S. M. S. Kazmi, L. M. Richards, C. J. Schrandt, M. A. Davis, and A. K. Dunn, "Expanding applications, accuracy, and interpretation of laser speckle contrast imaging of cerebral blood flow," J. Cereb. Blood Flow Metab., vol. 35, no. 7, pp. 1076-1084, 2015.

[31] J. Senarathna, S. Member, A. Rege, N. Li, and N. V. Thakor, "Laser speckle contrast imaging: Theory, instrumentation and applications," IEEE Rev. Biomed. Eng., vol. 6, no. 1, pp. 99-110, 2013.

[32] D. D. Duncan, S. J. Kirkpatrick, and J. C. Gladish, "What is the proper statistical model for laser speckle flowmetry?" Proc. SPIE, vol. 6855, pp. 685502-1-685502-7, 2008.

[33] K. Basak, G. Dey, M. Mahadevappa, M. Mandal, and P. Dutta, "In vivo laser speckle imaging by adaptive contrast computation for microvasculature assessment," Opt. Lasers Eng., vol. 62, pp. 87-94, 2014.

[34] S. Kazmi, E. Faraji, M. Davis, Y.-Y. Huang, X. Zhang, and A. Dunn, "Flux or speed? Examining speckle contrast imaging of vascular flows," Biomed. Opt. Exp., vol. 6, no. 7, pp. 2588-2608, 2015.

[35] J. C. Ramirez-San-Juan, R. Ramos-García, I. Guizar-Iturbide, G. Martínez-Niconoff, and B. Choi, "Impact of velocity distribution assumption on simplified laser speckle imaging equation." Opt. Exp., vol. 16, no. 5, pp. 3197-3203, 2008.

[36] A. K. Dunn, "Laser speckle contrast imaging of cerebral blood flow," Ann. Biomed. Eng., vol. 40, no. 2, pp. 367-377, 2012.

[37] R. Bi, J. Dong, C. L. Poh, and K. Lee, "Optical methods for blood perfusion measurement-Theoretical comparison among four different modalities," J. Opt. Soc. Amer. A, vol. 32, no. 5, pp. 860-866, 2015.

[38] A. Khalil, A. Humeau-Heurtier, G. Mahé, and P. Abraham, "Laser speckle contrast imaging: age-related changes in microvascular blood flow and correlation with pulse-wave velocity in healthy subjects," J. Biomed. Opt., vol. 20, no. 5, p. 051010, 2015.

[39] S. M. S. Kazmi, R. K. Wu, and A. K. Dunn, "Evaluating multi-exposure speckle imaging estimates of absolute autocorrelation times," Opt. Lett., vol. 40, no. 15, pp. 3643-3646, 2015.

[40] A. Parthasarathy, S. Kazmi, and A. Dunn, "Quantitative imaging of ischemic stroke through thinned skull in mice with multi exposure speckle imaging," Biomed. Opt. Exp., vol. 1, no. 1, pp. 246-259, 2010. 
[41] Y. Atchia, H. Levy, S. Dufour, and O. Levi, "Rapid multiexposure in vivo brain imaging system using vertical cavity surface emitting lasers as a light source," Appl. Opt., vol. 52, no. 7, pp. C64-C71, 2013.

[42] S. M. Kazmi, S. Balial, and A. K. Dunn, "Optimization of camera exposure durations for multi-exposure speckle imaging of the microcirculation," Biomed. Opt. Exp., vol. 5, no. 7, pp. 2157-2171, 2014.

[43] H. Cheng and T. Q. Duong, "Simplified laser-speckle-imaging analysis method and its application to retinal blood flow imaging," Opt. Lett., vol. 32, no. 15, pp. 2188-2190, 2007.

[44] T. Smausz, D. Zölei, and B. Hopp, "Real correlation time measurement in laser speckle contrast analysis using wide exposure time range images," Appl. Opt., vol. 48, no. 8, pp. 1425-1429, 2009.

[45] O. B. Thompson and M. K. Andrews, "Spectral density and tissue perfusion from speckle contrast measurements," Proc. SPIE, vol. 6847, pp. 68472D-1-68472D-7, 2008.

[46] D. Zölei, T. Smausz, B. Hopp, and F. Bari, "Self-tuning laser speckle contrast analysis based on multiple exposure times with enhanced temporal resolution," J. Eur. Opt. Soc., vol. 8, 2013.

[47] P.-A. Lemieux and D. J. Durian, "Investigating non-Gaussian scattering processes by using nth-order intensity correlation functions," J. Opt. Soc. Amer. A, vol. 16, no. 7, pp. 1651-1664, 1999.

[48] A. K. Dunn, H. Bolay, M. A. Moskowitz, and D. A. Boas, "Dynamic imaging of cerebral blood flow using laser speckle," J. Cereb. Blood Flow Metab., vol. 21, no. 3, pp. 195-201, 2001

[49] P. Zakharov, A. Völker, A. Buck, B. Weber, and F. Scheffold, "Quantitative modeling of laser speckle imaging," Opt. Lett., vol. 31, no. 23, pp. 3465-3467, 2006

[50] A. Mazhar, D. Cuccia, T. Rice, S. Carp, A. Durkin, D. Boas, B. Choi, and B. Tromberg, "Laser speckle imaging in the spatial frequency domain," Biomed. Opt. Exp., vol. 2, no. 6, pp. 1553-1563, 2011.

[51] A. E. Ennos, "Speckle interferometry," in Laser Speckle Relat. Phenom., ser. Topics in Applied Physics, vol. 9. New York, NY, USA: Springer, 1975 , ch. 6, pp. 203-253.

[52] K. Basak, M. Manjunatha, and P. Dutta, "Review of laser speckle-based analysis in medical imaging," Med. Bol. Eng. Comput., vol. 50, no. 6, pp. 547-58, Jun. 2012.

[53] J. D. Briers, "Laser Doppler and time-varying speckle: A reconciliation," J. Opt. Soc. Amer. A, vol. 13, no. 2, pp. 345-350, 1996.

54] D. Magatti, A. Gatti, and F. Ferri, "Three-dimensional coherence of light speckles: Experiment," Phys. Rev. A-At. Mol. Opt. Phys., vol. 79, pp. 1-11, 2009.

[55] O. B. Thompson and M. K. Andrews, "Tissue perfusion measurements: Multiple-exposure laser speckle analysis generates laser Doppler-like spectra," J. Biomed. Opt., vol. 15, no. 2, pp. 027095-1-027095-7, 2010.

[56] J. C. Ramirez-San-Juan, E. Mendez-Aguilar, N. Salazar-Hermenegildo, A. Fuentes-Garcia, R. Ramos-Garcia, and B. Choi, "Effects of speckle/pixel size ratio on temporal and spatial speckle-contrast analysis of dynamic scattering systems: Implications for measurements of blood-flow dynamics," Biomed. Opt. Exp., vol. 4, no. 10, pp. 1883-1889, 2013.

[57] S. J. Kirkpatrick, D. D. Duncan, and E. M. Wells-Gray, "Detrimental effects of speckle-pixel size matching in laser speckle contrast imaging," Opt. Lett., vol. 33, no. 22, pp. 2886-2888, 2008.

[58] C. P. Valdes, H. M. Varma, A. K. Kristoffersen, T. Dragojevic, J. P. Culver, and T. Durduran, "Speckle contrast optical spectroscopy, a non-invasive, diffuse optical method for measuring microvascular blood flow in tissue," Biomed. Opt. Exp., vol. 5, no. 8, pp. 2769-2784, 2014.

[59] J. Qiu, Y. Li, Q. Huang, Y. Wang, and P. Li, "Correcting speckle contrast at small speckle size to enhance signal to noise ratio for laser speckle contrast imaging," Opt. Exp., vol. 21, no. 23, pp. 255-265, 2013.

[60] J. Bennett, "Polarization," in Handbook of Optics, 3rd ed., M. Bass, Ed. New York, NY, USA: McGraw-Hill, 2001, ch. 12, pp. 12.3-12.30.

[61] D. Brewster, "On the laws which regulate the polarisation of light by reflexion from transparent bodies," Philos. Trans. R. Soc. London, vol. 105, pp. 125-159, Jan. 1815.

[62] H. Li, Q. Liu, H. Lu, Y. Li, H. F. Zhang, and S. Tong, "Directly measuring absolute flow speed by frequency-domain laser speckle imaging," Opt. Exp., vol. 22, no. 17, pp. 21079-21087, 2014.

[63] S. Sun, B. R. Hayes-Gill, D. He, Y. Zhu, and S. P. Morgan, "Multiexposure laser speckle contrast imaging using a high frame rate CMOS sensor with a field programmable gate array," Opt. Lett., vol. 40, no. 20, pp. 4587-4590, 2015 .

[64] S. Yuan, A. Devor, D. Boas, and A. Dunn, "Determination of optimal exposure time for imaging of blood flow changes with laser speckle contrast imaging." Appl. Opt., vol. 44, no. 10, pp. 1823-1830, 2005.
[65] H. M. Varma, C. P. Valdes, A. K. Kristoffersen, J. P. Culver, and T. Durduran, "Speckle contrast optical tomography: A new method for deep tissue three-dimensional tomography of blood flow," Biomed. Opt. Exp., vol. 5, no. 4, pp. 1275-1289, 2014.

[66] P. Zakharov, F. Scheffold, and B. Weber, "Laser speckle analysis synchronised with cardiac cycle," in Proc. Eur. Conf. Biomed. Opt., 2015 pp. 954008-1-954008-9.

[67] O. Yang, D. Cuccia, and B. Choi, "Real-time blood flow visualization using the graphics processing unit," J. Biomed. Opt., vol. 16, no. 1 pp. 016009-1-016009-14, 2011.

[68] M. Nemati, L. G. Paroni, N. Bhattacharya, and H. P. Urbach, "Fluid pulsation detection in presence of induced motion artifacts using speckle techniques," AIP Conf. Proc., vol. 1600, pp. 215-222, 2014.

[69] L. M. Richards, S. M. S. Kazmi, J. L. Davis, K. E. Olin, and A. K. Dunn, "Low-cost laser speckle contrast imaging of blood flow using a webcam," Biomed. Opt. Exp., vol. 4, no. 10, pp. 2269-2283, Jan. 2013.

[70] K. R. Forrester, C. Stewart, J. Tulip, C. Leonard, and R. C. Bray, "Comparison of laser speckle and laser Doppler perfusion imaging: Measurement in human skin and rabbit articular tissue," Med. Biol. Eng. Comput., vol. 40, no. 6, pp. 687-697, Nov. 2002.

[71] C. Scully, "Detection of spatial and temporal interactions in renal autoregulation dynamics," Ph.D. dissertation, Dept. Biomed Eng., Worcester Polytechnic Inst., Worcester, MA, USA, pp. 28-72, 2013.

[72] A. Rege, N. Thakor, K. Rhie, and A. Pathak, "In vivo laser speckle imaging reveals microvascular remodeling and hemodynamic changes during wound healing angiogenesis," Angiogenesis, vol. 15, no. 1, pp. 87-98, 2012.

[73] H. Fujii, T. Asakura, K. Nohira, Y. Shintomi, and T. Ohura, "Blood flow observed by time-varying laser speckle," Opt. Lett., vol. 10, no. 3, pp. 104-106, 1985.

[74] H. Fujii, K. Nohira, Y. Yamamoto, H. Ikawa, and T. Ohura, "Evaluation of blood flow by laser speckle image sensing. Part 1," Appl. Opt., vol. 26, no. 24, pp. 5321-5325, 1987

[75] Y. Tamaki, M. Araie, E. Kawamoto, S. Eguchi, and H. Fujii, "Noncontact, two-dimensional measurement of retinal microcirculation using laser speckle phenomenon," Invest. Ophthalmol. Vis. Sci., vol. 35, no. 11, pp. 3825-3834, Oct. 1994

[76] G. Mahé, A. Humeau-Heurtier, S. Durand, G. Leftheriotis, and P. Abraham, "Assessment of skin microvascular function and dysfunction with laser speckle contrast imaging," Circ. Cardiovasc. Imag., vol. 5, no. 1, pp. 155-63, Jan. 2012.

[77] A. Humeau-Heurtier, E. Guerreschi, P. Abraham, and G. Mahé, "Relevance of laser doppler and laser speckle techniques for assessing vascular function: State of the art and future trends," IEEE Trans. Biomed. Eng., vol. 60 , no. 3 , pp. 659-666, Mar. 2013.

[78] O. Thompson, J. Bakker, C. Kloeze, E. Hondebrink, and W. Steenbergen, "Experimental comparison of perfusion imaging systems using multiexposure laser speckle, single-exposure laser speckle, and full-field laser Doppler," Dyn. Fluctuations Biomed. Photon. IX, vol. 8222, pp. 822204 $1-822204-8,2012$

[79] H. Cheng, Q. Luo, S. Zeng, S. Chen, J. Cen, and H. Gong, "Modified laser speckle imaging method with improved spatial resolution," J. Biomed. Opt., vol. 8, no. 3, pp. 559-564, 2003.

[80] D. D. Duncan, S. J. Kirkpatrick, and R. K. Wang, "Statistics of loca speckle contrast." J. Opt. Soc. Amer. A. Opt. Image Sci. Vis., vol. 25, no. 1, pp. 9-15, 2008.

[81] H. Cheng, Y. Yan, and T. Q. Duong, "Temporal statistical analysis of laser speckle images and its application to retinal blood-flow imaging." Opt. Exp., vol. 16, no. 14, pp. 10214-10219, 2008.

[82] J. Ramirez-San-Juan, C. Regan, B. Coyotl-Ocelotl, and B. Choi, "Spatial versus temporal laser speckle contrast analyses in the presence of static optical scatterers," J. Biomed. Opt., vol. 19, no. 10, p. 106009, 2014.

[83] P. Li, Y. Li, H. He, Y. Tang, and M. Chen, "Methods on improving sampling depth of laser speckle contrast imaging of blood flow," presented at the Asia Communication Photonics Conf., Beijing, China, 2013, Paper AF1J.4

[84] A. Rege, J. Senarathna, N. Li, and N. V. Thakor, "Anisotropic processing of laser speckle images improves spatiotemporal resolution," IEEE Trans. Biomed. Eng., vol. 59, no. 5, pp. 1272-1280, May 2012.

[85] D. Duncan and S. Kirkpatrick, "Spatio-temporal algorithms for processing laser speckle imaging data," Proc. SPIE, vol. 6858 pp. 685802-1-685802-6, 2008 
[86] J. Qiu, P. Li, W. Luo, H. Zhang, and Q. Luo, "Spatiotemporal laser speckle contrast analysis for blood flow imaging with maximized speckle contrast," J. Biomed. Opt., vol. 15, no. 1, pp. 016003-1-016003-5, 2010.

[87] N. Li, X. Jia, K. Murari, R. Parlapalli, A. Rege, and N. V. Thakor, "High spatiotemporal resolution imaging of the neurovascular response to electrical stimulation of rat peripheral trigeminal nerve as revealed by in vivo temporal laser speckle contrast," J. Neurosci. Methods, vol. 176, no. 2, pp. 230-236, 2009.

[88] J. Allen and K. Howell, "Microvascular imaging: Techniques and opportunities for clinical physiological measurements," Physiol. Meas. vol. 35, pp. R91-R141, 2014.

[89] G. Armitage, K. Todd, A. Shuaib, and I. Winship, "Laser speckle contrast imaging of collateral blood flow during acute ischemic stroke," J. Cereb. Blood Flow Metabolism, vol. 30, no. 8, pp. 1432-1436, Aug. 2010.

[90] Z. Wang, W. Luo, P. Li, J. Qiu, and Q. Luo, "Acute hyperglycemia compromises cerebral blood flow following cortical spreading depression in rats monitored by laser speckle imaging," J. Biomed. Opt., vol. 13, no. 6, pp. 64023-64026, 2008

[91] A. B. Parthasarathy, E. L. Weber, L. M. Richards, D. J. Fox, and A. K. Dunn, "Laser speckle contrast imaging of cerebral blood flow in humans during neurosurgery: A pilot clinical study," J. Biomed. Opt., vol. 15, no. 6, pp. 66030-66038, 2010.

[92] F. Domoki, D. Zölei, O. Oláh, V. Tóth-Szki, B. Hopp, F. Bari, and T. Smausz, "Evaluation of laser-speckle contrast image analysis techniques in the cortical microcirculation of piglets," Microvasc. Res., vol. 83, no. 3, pp. 311-317, 2012.

[93] I. Kofman and D. Abookasis, "Dual-wavelength laser speckle imaging for monitoring brain metabolic and hemodynamic response to closed head traumatic brain injury in mice," J. Biomed. Opt., vol. 20, no. 10, p. $106009,2015$.

[94] A. I. Srienc, Z. L. Kurth-Nelson, and E. A. Newman, "Imaging retinal blood flow with laser speckle flowmetry," Front. Neuroenergetics, vol. 2, pp. 1-10, 2010.

[95] Y. Shiga, M. Shimura, T. Asano, S. Tsuda, Y. Yokoyama, N. Aizawa, K. Omodaka, M. Ryu, S. Yokokura, T. Takeshita, and T. Nakazawa, "The influence of posture change on ocular blood flow in normal subjects, measured by laser speckle flowgraphy," Curr. Eye Res., vol. 38, no. 6, pp. 691-698, May 2013

[96] A. Ponticorvo, D. Cardenas, A. K. Dunn, D. Tso, and T. Q. Duong, "Laser speckle contrast imaging of blood flow in rat retinas using an endoscope," J. Biomed. Opt., vol. 18, no. 9, p. 90501, 2013.

[97] B. Ruaro, A. Sulli, E. Alessandri, C. Pizzorni, G. Ferrari, and M. Cutolo, "Laser speckle contrast analysis: A new method to evaluate peripheral blood perfusion in systemic sclerosis patients," Ann. Rheumatic Dis., vol. 73, pp. 1181-1185, 2013.

[98] A. Sulli, B. Ruaro, and M. Cutolo, "Evaluation of blood perfusion by laser speckle contrast analysis in different areas of hands and face in patients with systemic sclerosis," Ann. Rheumatic Dis., vol. 73, no. 11, pp. 2059-2061, 2014.

[99] R. T. Domsic, C. Dezfulian, A. Shoushtari, D. Ivanco, E. Kenny, C. K. Kwoh, T. A. Medsger Jr, and H. C. Champion, "Endothelial dysfunction is present only in the microvasculature and microcirculation of early diffuse systemic sclerosis patients," Clin. Exp. Rheumatol., vol. 32, no. Suppl 86, p. S-154-S-160, 2014.

[100] J. D. Pauling, J. A. Shipley, D. J. Hart, A. McGrogan, and N. J. McHugh, "Use of laser speckle contrast imaging to assess digital microvascular function in primary raynaud phenomenon and systemic sclerosis: A comparison using the raynaud condition score diary," J. Rheumatol., vol. 42, no. 7 , pp. $1163-1168,2015$.

[101] C. Puissant, P. Abraham, S. Durand, A. Humeau-Heurtier, S. Faure, G. Lefthériotis, P. Rousseau, and G. Mahé, "Reproducibility of noninvasive assessment of skin endothelial function using laser doppler flowmetry and laser speckle contrast imaging," PLoS One, vol. 8, no. 4, p. e61320, 2013.

[102] I. Cordovil, G. Huguenin, G. Rosa, A. Bello, O. Khler, R. de Moraes and E. Tibiri, "Evaluation of systemic microvascular endothelial function using laser speckle contrast imaging," Microvascular Res., vol. 83, no. 3, pp. 376-379, 2012.

[103] P. Vaz, T. Pereira, E. Figueiras, C. Correia, A. Humeau-Heurtier, and J. Cardoso, "Which wavelength is the best for arterial pulse waveform extraction using laser speckle imaging?" Biomed. Signal Process. Control, vol. 25, pp. 188-195, 2016.

[104] Y. Beiderman, I. Horovitz, N. Burshtein, M. Teicher, J. Garcia, V. Mico, and Z. Zalevsky, "Remote estimation of blood pulse pressure via tem- poral tracking of reflected secondary speckles pattern," J. Biomed. Opt., vol. 15, no. 6, pp. 0617017-1-0617017-7, 2010.

[105] M. Nemati, G. Loozen, N. van der Wekken, G. van de Belt, H. Urbach, N. Bhattacharya, and S. Kenjeres, "Application of full field optical studies for pulsatile flow in a carotid artery phantom," Biomed. Opt. Exp. vol. 6, no. 10, pp. 4037-4050, 2015.

[106] C. Regan, B. Yang, K. Mayzel, J. Ramirez-San-Juan, P. Wilder-Smith, and B. Choi, "Fiber-based laser speckle imaging for the detection of pulsatile flow," Lasers Surg. Med., vol. 47, no. 6, pp. 520-525, 2015.

[107] M. Nemati, C. Presura, H. Urbach, and N. Bhattacharya, "Dynamic light scattering from pulsatile flow in the presence of induced motion artifacts," Biomed. Opt. Exp., vol. 5, no. 7, pp. 2145-2156, 2014.

[108] N. Sujatha and A. Banerjee, "An experimental model for minimizing errors in laser speckle contrast imaging for microcirculation analysis," in Proc. Int. Conf. Exp. Mech., 2015, vol.9302, pp. 93021R-1-93021R-6.

[109] M. Davis, S. M. S. Kazmi, and A. K. Dunn, "Imaging depth and multiple scattering in laser speckle contrast imaging," J. Biomed. Opt., vol. 19, no. 8, p. $086001,2014$.

[110] Y. Ji, Z. Chao, Y. Zhang, Z. Wu, and P. Miao, "Effects of scattering particles' concentration in laser speckle contrast imaging," in Proc. IEEE 7th Int. Conf. Biomed. Eng. Informat., 2014, pp. 56-60.

[111] Y. Shiga, T. Asano, H. Kunikata, F. Nitta, H. Sato, T. Nakazawa, and M. Shimura, "Relative flow volume, a novel blood flow index in the human retina derived from laser speckle flowgraphy," Investig. Opthalmology Vis. Sci., vol. 55, no. 6, pp. 3899-3904, 2014

[112] K. R. Forrester, J. Tulip, C. Leonard, C. Stewart, and R. C. Bray, “A laser speckle imaging technique for measuring tissue perfusion," IEEE Trans. Biomed. Eng., vol. 51, no. 11, pp. 2074-2084, Nov. 2004.

[113] S. M. S. Kazmi, A. B. Parthasarthy, N. E. Song, T. A. Jones, and A. K. Dunn, "Chronic imaging of cortical blood flow using MultiExposure Speckle Imaging," J. Cereb. Blood Flow Metab., vol. 33, no. 6, pp. 798-808, 2013.

[114] Z. Luo, Z. Wang, Z. Yuan, C. Du, and Y. Pan, "Optical coherence Doppler tomography quantifies laser speckle contrast imaging for blood flow imaging in the rat cerebral cortex," Opt. Lett., vol. 33, no. 10, pp. 11561158, 2008.

[115] J. ODoherty, P. McNamara, N. Clancy, J. Enfield, and M. Leahy, "Comparison of instruments for investigation of microcirculatory blood flow and red blood cell concentration," J. Biomed. Opt., vol. 14, no. 3, p. 34025,2009

[116] I. Fredriksson, M. Larsson, and T. Strömberg, "Measurement depth and volume in laser Doppler flowmetry," Microvasc. Res., vol. 78, no. 1, pp. 4-13, 2009

[117] R. Bi, J. Dong, and K. Lee, "Deep tissue flowmetry based on diffuse speckle contrast analysis," Opt. Lett., vol. 38, no. 9, pp. 1401-1403, 2013.

[118] H. He, Y. Tang, F. Zhou, J. Wang, Q. Luo, and P. Li, "Lateral laser speckle contrast analysis combined with line beam scanning illumination to improve the sampling depth of blood flow imaging," Opt. Lett., vol. 37, no. 18 , pp. 3774-3776, Sep. 2012.

[119] I. Sigal, R. Gad, A. M. Caravaca-Aguirre, Y. Atchia, D. B. Conkey, R. Piestun, and O. Levi, "Laser speckle contrast imaging with extended depth of field for in-vivo tissue imaging," Biomed. Opt. Exp., vol. 5 , no. 1 , pp. $123-135,2014$

[120] R. Bi, J. Dong, and K. Lee, "Multi-channel deep tissue flowmetry based on temporal diffuse speckle contrast analysis," Opt. Exp., vol. 21, no. 19, pp. 22854-22861, 2013.

[121] M. M. Gonik, A. B. Mishin, and D. A. Zimnyakov, "Visualization of blood microcirculation parameters in human tissues by time-integrated dynamic speckles analysis," Ann. New York Acad. Sci., vol. 972, no. 1, pp. 325-330, Oct. 2002.

[122] T. Durduran and A. G. Yodh, "Diffuse correlation spectroscopy for noninvasive, micro-vascular cerebral blood flow measurement," Neuroimage vol. 85, pp. 51-63, 2014.

[123] P. Vaz, R. Couceiro, P. Carvalho, and J. Henriques, “An Automatic method for motion artifacts detection in photoplethysmographic signals referenced with electrocardiography data," in Proc. IEEE 7th Int. Conf. Biomed. Eng. Informat., 2014, pp. 704-708.

Authors' photographs and biographies not available at the time of publication. 